\title{
Síndrome de dificultad respiratoria aguda por compromiso pulmonar en malaria complicada: a propósito de un caso
}

\section{Acute respiratory distress syndrome due to pulmonary involvement in complicated malaria-case report}

Mary Luz Moreno Vega, MD ${ }^{1}$ ERnesto Santiago, MD. ${ }^{2}$

\section{Resumen}

La malaria es la enfermedad parasitaria más importante del ser humano y representa un problema de salud pública en más de 91 países. Mientras en la infección no complicada la mortalidad es cercana al 0,1\%, en malaria complicada se incrementa de forma gradual asociada principalmente con las complicaciones graves de la infección como malaria cerebral, anemia grave, dificultad respiratoria, insuficiencia renal y malaria grave del embarazo. La incidencia de las complicaciones pulmonares ha aumentado en las últimas décadas. Su forma clínica más grave es el síndrome de dificultad respiratoria aguda (SDRA), que tiene una elevada mortalidad a pesar de un abordaje terapéutico adecuado.

Se expone el caso de un paciente con malaria complicada por Plasmodium falciparum, quien cursó con hallazgos clínicos y radiológicos de SDRA en el contexto de compromiso pulmonar asociado.

Palabras clave: malaria, Plasmodium falciparum, síndrome de dificultad respiratoria aguda, neumonía.

\begin{abstract}
Malaria is the most important human being's parasitic disease, representing a public health challenge in more than 91 countries. While uncomplicated infection the mortality is close to $0.1 \%$, in complicated malaria it increases gradually associated, mainly, to the serious complications infection like cerebral malaria, severe anemia, difficulty of breathing, renal failure and severe pregnancy malaria. The incidence of pulmonary manifestations has increased in recent years. Acute respiratory distress syndrome (ADRS) is the most severe form within the pulmonary complications of malaria, with high mortality despite proper management. We present a clinical case of a patient with malaria complicated by Plasmodium falciparum with clinical and radiological findings of ARDS in the context of associated pulmonary involvement.
\end{abstract} monia.

Keywords: Malaria, Plasmodium falciparum, acute respiratory distress syndrome, pneu-
${ }^{1}$ Residente de segundo año de Medicina Interna. Universidad Libre seccional Atlántico.

${ }^{2}$ Internista neumólogo. Docente del posgrado de Medicina interna adscrito a la Universidad Libre, Universidad metropolitana.

Autor de correspondencia

Mary Luz Moreno Vega. Correo electrónico:malu1486@live.com.

Recibido: 4/10/2018. Aceptado:

4/11/2018. 


\section{Introducción}

La malaria es la enfermedad parasitaria más importante del ser humano, con transmisión activa en 91 países a través de la picadura del mosquito hembra Anopheles infectado, que causa aproximadamente 1200 muertes cada día (1). Representa para Colombia un problema prioritario de salud pública debido al aumento en la transmisión en determinadas regiones del país y la inversión en la proporción del parásito implicado, predominando la infección por Plasmodium falciparum (2). El cuadro clínico comprende desde una infección no complicada (en la que el paciente puede digerir alimentos y medicinas) hasta la malaria complicada (una vez ocurre disfunción de órganos vitales o cuando la proporción total de eritrocitos infectados se incrementa a más de $2 \%)(1,3)$. Mientras que en la infección no complicada la mortalidad es cercana al 0,1 $\%$, en la infección complicada se incrementa de forma gradual asociada principalmente con las complicaciones graves de la infección como malaria cerebral, anemia grave, dificultad respiratoria, insuficiencia renal y malaria grave del embarazo $(3,4)$.

La alteración pulmonar más importante atribuible directamente a $P$. falciparum es el edema pulmonar no cardiogénico y su evolución más temida es a un síndrome de dificultad respiratoria aguda (SDRA) $(3,4)$.

La incidencia de las complicaciones pulmonares ha aumentado en las últimas décadas. Algunos estudios describen la presencia de síntomas respiratorios hasta en un $4 \%-18 \%$ de los pacientes adultos con malaria por $P$. falciparum; y en estudios post mortem, 21 $\%-23 \%$ de los pacientes no inmunes desarrolla edema pulmonar $(4,5)$. A pesar de este reconocimiento, los profesionales médicos pueden estar poco familiarizados con la presentación y diagnóstico de las complicaciones pulmonares en malaria, lo cual contribuye al retraso en el tratamiento y una mayor mortalidad.

Se expone el caso de un paciente con malaria complicada por $P$. falciparum a quien se le atribuye SDRA como manifestación del compromiso pulmonar.

\section{Reporte de caso}

Paciente de sexo masculino de 39 años quien consultó por un cuadro de 6 días de evolución caracteriza- do por episodios febriles, no cuantificados, precedidos de escalofríos, asociados con cefalea, dolor epigástrico urente irradiado a los hipocondrios y poliartralgias sin efusión articular. En las 48 horas previas al ingreso presentó disnea moderada y tos seca. Antecedente de desplazamiento a Bahía Solano, Chocó, Colombia, para la práctica de pesca recreativa hace 2 semanas. Sin antecedentes patológicos o exposicionales a destacar.

Ingresó al servicio de urgencias en regular estado general, tinte ictérico generalizado, taquipneico, sin tolerancia al decúbito dorsal, saturación arterial de oxígeno $\left(\mathrm{SaO}_{2}\right): 90 \%$, (fracción inspirada de oxígeno $\left[\mathrm{FIO}_{2}\right]$ ambiental), tensión arterial (TA): 120/80 mm Hg, frecuencia cardíaca (FC): 90 latidos por minuto (lpm), frecuencia respiratoria (FR): 24 respiraciones por minuto (rpm), temperatura $\left(\mathrm{T}^{\circ}\right): 37,5^{\circ} \mathrm{C}$. Escleras ictéricas, ruidos cardíacos rítmicos, sin soplos, murmullo vesicular disminuido en ambas bases pulmonares. Abdomen depresible, hepatomegalia, sin signos de irritación peritoneal. Los resultados de los exámenes iniciales se presentan en la Tabla 1. Radiografía de tórax en proyección posteroanterior (PA) y lateral que para este momento se interpretó como normal (Figura 1).

A la luz de los hallazgos clínicos y paraclínicos se planteó un cuadro clínico compatible con malaria complicada por $P$. falciparum, se admitió a hospitalización y se inició una terapia antimalárica oral con arteméter/ lumefantrina. Tres horas después del ingreso, el paciente cursaba con evolución tórpida con dificultad respiratoria y crépitos en la base derecha; se le realizó un control radiológico en el que se apreció una ocupación alveolar en el lóbulo medio e inferior derecho (Figura 2); los gases arteriales revelaron un trastorno mixto (alcalosis respiratoria y acidosis metabólica) e hipoxemia moderada (Tabla 1). Se consideró un compromiso pulmonar asociado con el cuadro ya configurado de malaria complicada, por lo cual se trasladó a la unidad de cuidado intensivo (UCI) donde, a su ingreso, se indicó el inicio de ventilación mecánica no invasiva con presión positiva en el contexto de insuficiencia respiratoria con hipoxemia, con lo cual se obtuvo una evolución favorable y se logró la descontinuación de esta a las 48 horas.

Presentó un alza térmica al tercer día de la admisión en la UCI, por lo cual se ordenó el control imagenológico con tomografía axial computarizada (TAC) 
de tórax, que evidenció un derrame pleural bilateral y extensas áreas densas de broncograma aéreo en la língula, segmento medial del lóbulo medio y segmento apical del lóbulo superior derecho (Figura 3). Se planteó como un diagnóstico diferencial del SDRA secundario a malaria, un proceso neumónico asociado con el cuidado de la salud y se decidió asociar la terapia an- timicrobiana con cefalosporina de cuarta generación, con evolución satisfactoria, por lo cual se trasladó a piso, donde completó esquema antimicrobiano durante 7 días, para posteriormente egresar de la institución. Se le realizó un control radiológico a las 2 semanas del egreso en el que se resaltó la resolución completa de las lesiones previamente descritas (Figura 4).

Tabla 1. Resultados de exámenes

\begin{tabular}{|c|c|}
\hline Paraclínicos & Resultados \\
\hline $\mathrm{Hb}$, Hcto & $12,3 \mathrm{~g} / \mathrm{dL} ; 34,3 \%$ \\
\hline Leucocitos, plaquetas & $7,540 / \mathrm{mm}^{3}, \mathrm{~N}: 74,6 \%, \mathrm{~L}: 20,2 \%, \mathrm{E}: 0,5 \%$ \\
\hline Creatinina; BUN & $37,000 / \mathrm{mm}^{3}$ \\
\hline Bilirrubina & $0,99 \mathrm{mg} / \mathrm{dL} ; 16,2 \mathrm{mg} / \mathrm{dL}$ \\
\hline & $\begin{array}{l}\text { Total: } 6,45 \mathrm{mg} / \mathrm{dL} \text {; fracción directa: } 6,20 \mathrm{mg} / \mathrm{dL} \text {; fracción indirecta: 0,25 mg/dL } \\
15,7 \mathrm{~s} ; 33,9 \mathrm{~s}\end{array}$ \\
\hline TP; TTP & $238 \mathrm{U} / \mathrm{L}$ \\
\hline Fosfatasa alcalina & TGO: 228 U/L; TGP: 207 U/L \\
\hline Transaminasas & Positivo para $P$. falciparum; recuento $132200 \mathrm{p} / \mu \mathrm{L}$ \\
\hline $\begin{array}{l}\text { Gota gruesa } \\
\text { Gases arteriales }\end{array}$ & $\begin{array}{l}\mathrm{pH}: 7,46, \mathrm{SaO}_{2}: 88,3 \%, \mathrm{pCO}_{2}: 19,6 \mathrm{~mm} \mathrm{Hg}, \mathrm{pO}_{2}: 52,2 \mathrm{~mm} \mathrm{Hg}, \mathrm{HCO}_{3}: 13,9 \mathrm{mmol} / \mathrm{L}, \text { EBvt: }-7,1 \\
\mathrm{mmol} / \mathrm{L}, \mathrm{PaO}_{2} / \mathrm{FiO}_{2}: 247\end{array}$ \\
\hline
\end{tabular}

BUN: nitrógeno ureico sanguíneo; $\mathrm{Hb}$ : hemoglobina; $\mathrm{HCO}_{3}$ : bicarbonato; Hcto: hematocrito; $\mathrm{PaO}_{2} / \mathrm{FiO}_{2}$ : presión arterial de oxígeno/fracción inspirada de oxígeno; $\mathrm{pCO}_{2}$ : presión parcial de dióxido de carbono; $\mathrm{pO}_{2}$ : presión parcial de oxígeno; TGO: transaminasa glutámico oxalacética.; TGP: transaminasa glutámico pirúvica; TP: tiempo de protrombina; TTP: tiempo de tromboplastina parcial.
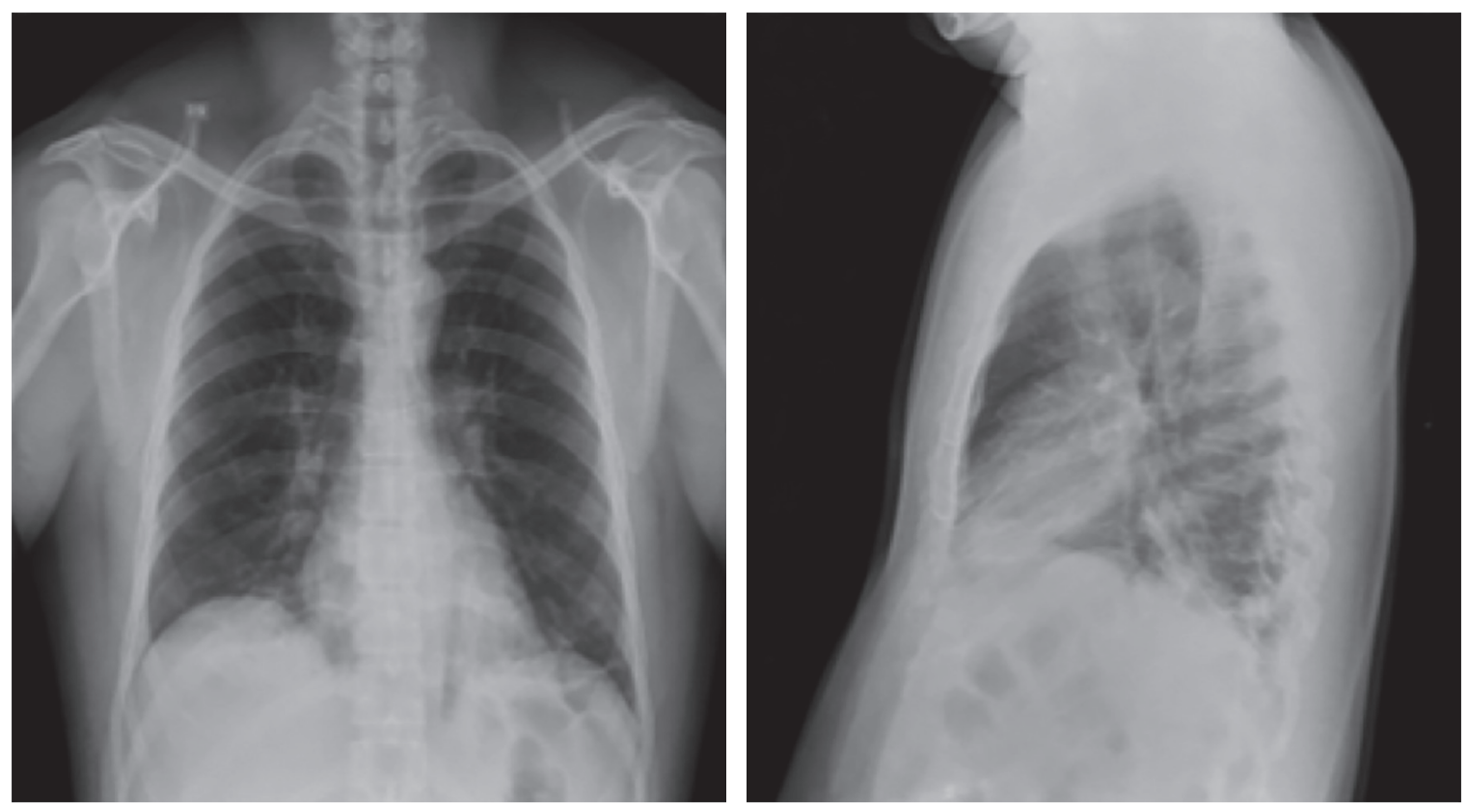

Figura 1. Radiografía de tórax en la que se aprecia en la proyección lateral el patrón de ocupación hacia el lóbulo medio e inferior derecho. 


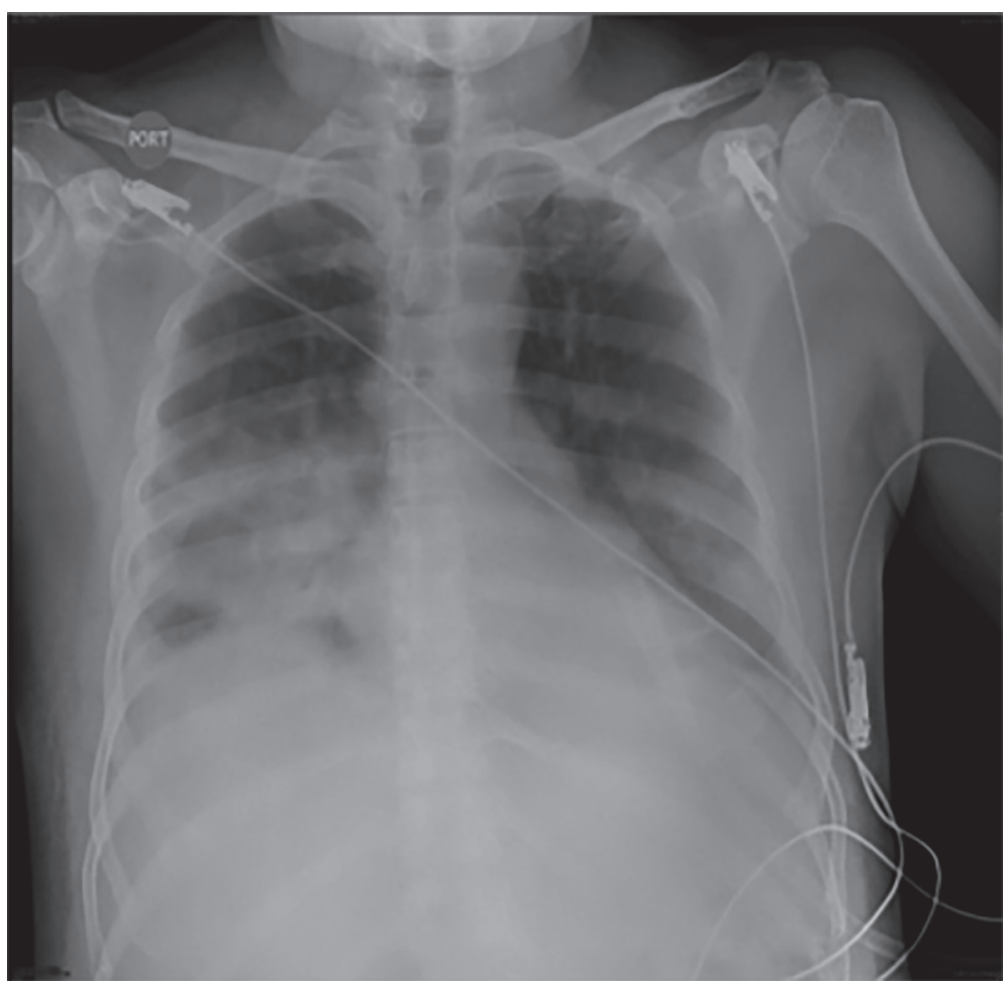

Figura 2. Radiografía de tórax de control a las 3 horas de ingreso. Se aprecia una franca ocupación alveolar en el lóbulo medio derecho.

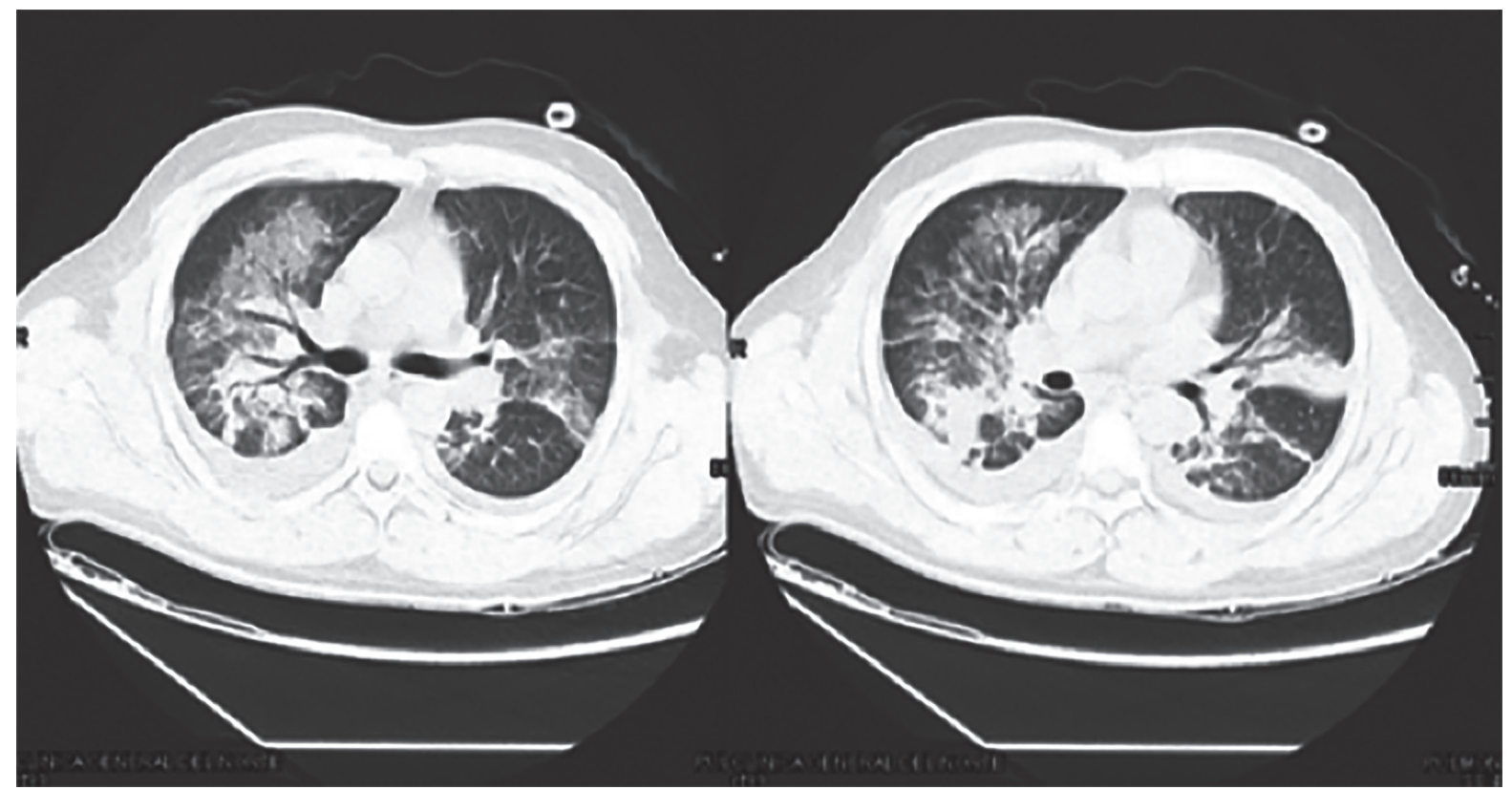

Figura 3. TAC de tórax. extensas áreas densas de broncograma aéreo en la língula, segmento medial del lóbulo medio y segmento apical del lóbulo superior derecho. 

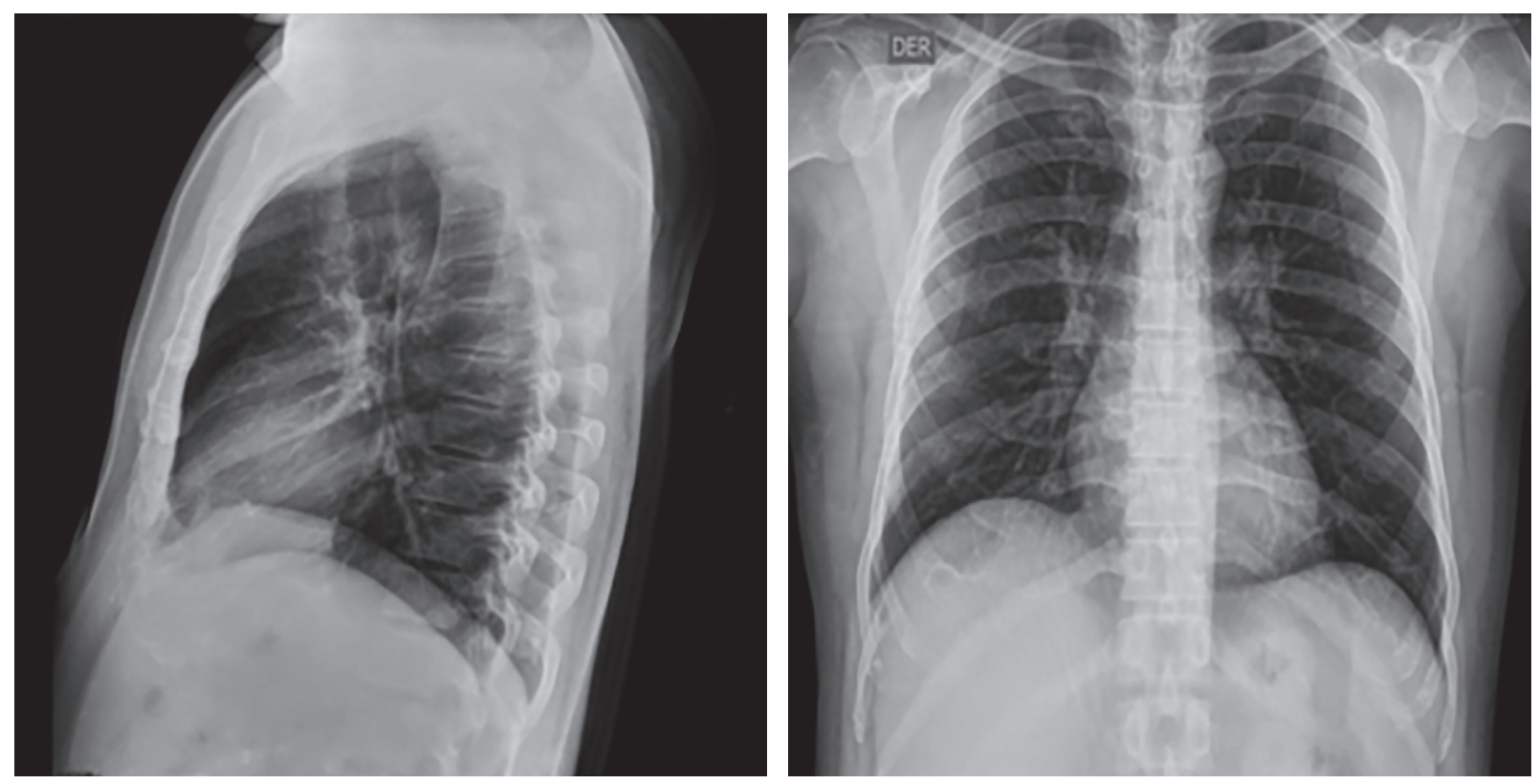

Figura 4. Radiografía de tórax a las 2 semanas con clara resolución de los signos radiológicos de compromiso inicial.

\section{Discusión}

El compromiso pulmonar en malaria suele manifestarse como una lesión pulmonar aguda (LPA), un SDRA o un edema pulmonar. También se han descrito otras expresiones clínicas como efusión pleural y edema intersticial aislado (6). En el presente caso, se documenta tanto un derrame pleural como un edema pulmonar en el curso de un SDRA, lo cual se corresponde con la evidencia en la literatura, en la que se describe hasta en $21 \%$-23\% de los pacientes no inmunes el desarrollo de edema pulmonar, y SDRA en $5 \%$ - $25 \%$ de las infecciones por $P$. falciparum y $1 \%-10 \%$ por Plasmodium vivax $(7,8)$. El edema pulmonar es el resultado de la respuesta inmunitaria frente al parásito con la destrucción de este y liberación de citocinas inflamatorias, que causan disfunción del endotelio y ruptura de la integridad de la barrera alveolocapilar $(1,3,4)$.

La incidencia del compromiso pulmonar ha aumentado en las últimas décadas y se han encontrado reportes de síntomas respiratorios hasta en un $4 \%$-18 $\%$ de los pacientes infectados $(4,5)$. Rajput y colaboradores mencionaron que el rango de edad en el que ocurren las complicaciones pulmonares oscila entre los 21 y 30 años, según los autores, en relación con la mayor proporción de jóvenes en la población, la falta de inmunidad contra la malaria y los viajes frecuentes a zonas endémicas $(1,9)$, como en el caso presentado, en el que se documentó un desplazamiento al Pacífico colombiano. Cabe resaltar que Chocó para el 2017, de acuerdo con el Sistema de Vigilancia en Salud Pública, aportó el 30,7 \% del total de la notificación nacional (2). La edad también se encuentra cerca al promedio descrito, así como era de esperar un cuadro florido ante la protección incompleta de la inmunidad adquirida y la especie aislada (1).

Dentro de los factores de riesgo para desarrollar compromiso pulmonar se describen:

1. Parasitemia elevada, aunque también se han documentado casos con parasitemia baja;

2. Instauración del cuadro clínico posterior al inicio de terapia antimalárica, aunque puede tener un curso más temprano, especialmente en el contexto de una malaria complicada;

3. Infección mixta ( $P$. vivax con P. falciparum) o superpuesta (dado que existe mayor riesgo de infecciones de origen bacteriano); $y$ 
4. Durante el curso clínico de una malaria complicada con disfunción multiorgánica, en el que existe una mayor claridad de esta relación causal (6).

En el caso expuesto, se pudo identificar un nexo epidemiológico al confirmar el desplazamiento a una zona endémica y como factores de riesgo la parasitemia alta, el curso de una disfunción multiorgánica y el compromiso temprano ya que, aunque la radiografía de tórax del inicio se interpretó como normal, en la revisión posterior de la proyección lateral se pudo apreciar una ocupación alveolar hacia el lóbulo inferior derecho (Figura 1).

El SDRA, como la complicación pulmonar más temida dentro del curso clínico de una malaria complicada (4), se caracteriza por una lesión pulmonar inflamatoria difusa que progresa rápidamente hacia el aumento de la permeabilidad vascular pulmonar, aumento del peso pulmonar e hipoxemia (10). Su diagnóstico se sustenta en el cumplimiento de los criterios de Berlín:

1. Inicio agudo de los síntomas (o empeoramiento de los síntomas no agudos) dentro de la primera semana del insulto clínico reconocido;

2. Hipoxemia documentada por una relación $\mathrm{PaO}_{2} /$ $\mathrm{FiO}_{2} \leq 300 \mathrm{~mm} \mathrm{Hg}$ con presión positiva al final de la espiración (PEEP) o presión positiva continua en la vía aérea (CPAP) de $\geq 5 \mathrm{~cm} \mathrm{H}_{2} \mathrm{O}$.

3. Imágenes de tórax con evidencia de opacidades bilaterales no atribuible por derrames, atelectasias o nódulos y no son de naturaleza cardiogénica (10).

Todos los criterios estaban presentes en el caso expuesto, en grado leve, teniendo en cuenta la clasificación por el nivel de hipoxemia que corresponde a leve, moderado o grave, con valores de $\mathrm{PaO}_{2} / \mathrm{FiO}_{2}$ entre 200 mm Hg y 300 mm Hg, 200 mm Hg y 100 mm Hg; y $\leq 100 \mathrm{~mm} \mathrm{Hg}$ respectivamente (10). Dado que las alteraciones radiológicas iniciales son mínimas y poco específicas, se recomienda una interpretación de forma seriada (11), como se planteó en el caso.
Respecto al manejo, hay que señalar que, tratándose de una emergencia médica, el pilar del tratamiento en el SDRA es la atención integral en el entorno de una UCI. Dentro de las opciones de tratamiento se plantea la ventilación mecánica mediante PEEP como una estrategia de protección pulmonar, ya sea con ventilación con volumen corriente bajo o ventilación con baja presión junto con maniobras de reclutamiento alveolar, aunque continúa la discusión sobre su eficacia en SDRA $(12,13)$; como se puede confrontar en el caso expuesto, el resultado es favorable.

En este caso se destacan aspectos relevantes como la confrontación del dilema de diagnósticos diferenciales como neumonía o acidosis metabólica frente al edema pulmonar del compromiso pulmonar en malaria complicada, dado que no hay evidencia definitiva de la existencia de neumonitis por malaria (14), llegando a recomendar incluso en zonas endémicas el cubrimiento antimicrobiano y antimalárico al mismo tiempo (14). Se tuvo la oportunidad de realizar un control radiológico a las 2 semanas del egreso, en el que se resalta la resolución radiológica, en menor tiempo de evolución según lo esperado frente al diagnóstico de neumonía (15); se considera que el cuadro clínico obedece a un SDRA por compromiso pulmonar en malaria complicada, con resolución favorable luego de la identificación del cuadro e instauración del manejo de forma temprana. Este es un caso relevante para no olvidar la consideración del compromiso pulmonar como parte de las manifestaciones letales en malaria.

\section{Referencias}

1. White NJ, Ashley EA. Paludismo. En: Kasper D, Fauci A, Longo D, et al (editores). Harrison principios de medicina interna. 20. ${ }^{\text {a }}$ edición. Chile: McGrawHill; 2018 [consultado el 10 de marzo de 2018]. Disponible en: https://accessmedicina.mhmedical.com/content.aspx?bookid=2461\&sectionid=209903288.

2. Zambrano P. Protocolo de vigilancia en salud pública. Malaria. Instituto Nacional de Salud [internet] 2014 [consultado el 1 de diciembre de 2018]. Disponible en: http://www.clinicamedihelp.com/documentos/protocolos/PRO\%20Malaria.pdf.

3. Fairhurst RM, Wellems TE. Género Plasmodium (malaria). En: Bennett JE, Dolin R, Blaser MJ (editores). Mandell, Douglas y Bennett. Enfermedades infecciosas. Principios y práctica. España: Elsevier; 2016. p. 3245-67. 
4. Cabezón Estévanez I., Górgolas Hernández-Mora M. Actualización de las complicaciones pulmonares de la malaria. Medicina Clínica. 2016; 146(8):354-58.

5. Anstey NM, Jacups SP, Cain T, Pearson T, Ziesing PJ, Fisher DA, et al. Pulmonary manifestations of uncomplicated falciparum and vivax malaria: cough, small airways obstruction, impaired gas transfer, and increased pulmonary phagocytic activity. J Infect Dis. 2002;185(9):1326-34.

6. Giraldo Sierra C, Blair Trujillo S, Tobón Castaño A. Complicaciones pulmonares en malaria. Infectio. 2005;8(4):279-92.

7. Bruneel F, Hocqueloux L, Alberti C, Wolff M, Chevret S, Bédos JP, et al. The clinical spectrum of severe imported falciparum malaria in the intensive care unit: report of 188 cases in adults. Am J Respir Crit Care Med. 2003;167(5):684-9.

8. Taylor WRJ, Hanson J, Turner GDH, White NJ, Dondorp AM. Respiratory manifestations of malaria. Chest. 2012;142(2):492-505.

9. Rajput R, Singh H, Singh S, Meena, Tiwari UC. Pulmonary manifestations in malaria. J Indian Med Assoc. 2000;98(10):612-4.
10. ARDS Definition Task Force, Ranieri VM, Rubenfeld GD, Thompson BT, Ferguson ND, Caldwell E, et al. Acute respiratory distress syndrome: the Berlin Definition. JAMA. 2012;307(23):2526-33.

11. Torres JR, Perez H, Postigo MM, Silva JR. Acute noncardiogenic lung injury in benign tertian malaria. Lancet. 1997;350(9070):31-2.

12. Sweet DD, Naismith A, Keenan SP, Sinuff T, Dodek PM. Missed opportunities for noninvasive positive pressure ventilation: a utilization review. J Crit Care. 2008;23(1):111-7.

13. Fan E, Brodie D, Slutsky AS. Acute Respiratory Distress Syndrome: Advances in Diagnosis and Treatment. JAMA. 2018;319(7):698-710.

14. O’Dempsey TJ, McArdle TF, Laurence B, Lamont IA, Todd JE, Greenwood BM. Overlap in the clinical features of pneumonia and malaria in African children. Transactions of the royal society of tropical medicine and higiene. 1993;87(6):662-5.

15. El Solh AA, Aquilina AT, Gunen H, Ramadan F. Radiographic resolution of community-acquired bacterial pneumonia in the elderly. J Am Geriatr Soc. 2004;52(2):224-9. 